\title{
Positron studies on reverse micellar systems of AOT/isooctane/water
}

\author{
M.F. FERREIRA MARQUES, C. LOPES GIL*, A.P. DE LIMA*, H.D. BURROWS** and \\ M. DA GRAÇA MIGUEL ${ }^{* *}$ \\ Instituto Superior de Engenharia, 3000 Coimbra, Portugal \\ * Departamento de Física da Universidade, 3000 Coimbra, Portugal \\ ** Departamento de Química da Universidade, 3000 Coimbra, Portugal
}

\begin{abstract}
AOT/Isooctane and water/AOT/Isooctane systems have been studied by positron annihilation techniques. Studies of the binary system show that AOT is a strong positron scavenger and support the idea of polydisperse aggregates. Results, for the ternary system, indicate the presence of monomers, even when aggregates are present, and show that Ps is in a water-free region. The effects of the addition of nitrobenzene, sodium nitrobenzoate and 4-phenylbutyric acid to the ternary system are studied. Ps reveals high reactivity towards nitrobenzene which is shown to be present both in isooctane and inside the aggregates.
\end{abstract}

\section{INTRODUCTION}

Positron annihilation techniques (PAT) provide a powerful method to study structures and changes in a variety of systems, including amphiphile solutions. Reports have been given of amphiphile aggregation in aqueous and non-aqueous solutions studied by PAT [1]. We concentrate here on two and three component systems involving AOT (sodium di-2-ethylhexylsulfosuccinate) in non-polar organic solvents. Although the type of aggregation is still not well defined, it is generally agreed that reversed micelles or microemulsions are formed [2].

If positrons $\left(\mathrm{e}^{+}\right)$are introduced into matter, they may combine with an electron to form a positronium atom, Ps. Ps can exist in two states, p-Ps, with 125 ps lifetime, and o-Ps, whose lifetime (ns time scale) is determined by pick-off reaction in the solvent. Studies of formation and decay of o-Ps can be particularly valuable in giving structural information on multiphase systems [3].

\section{EXPERIMENTAL}

AOT was dissolved in methanol, filtered with alumina and the solvent evaporated. Other reagents were of the purest grade available. Solutions were degassed using freeze-pump-thaw cycles. A $12 \mu \mathrm{Ci} 22 \mathrm{Na}$ positron source was introduced inside the reaction cell. The source was prepared by evaporation of carrier free ${ }^{22} \mathrm{NaCl}$ aqueous solution on a thin glass blade and further diffusion of the ${ }^{22} \mathrm{Na}$ into the glass. Positron lifetimes and Doppler lineshape parameter, $I_{V}$ (integrated over $\pm 2.0 \mathrm{mrad}$ around the peak) were measured. Time spectra were analysed assuming triexponential decay using the program POSITRONFIT [4], with the constraints $\tau_{1}(\mathrm{p}-\mathrm{Ps})=125 \mathrm{ps}$, and $\mathrm{I}_{1}=\mathrm{I}_{3} / 3$.

\section{RESULTS AND DISCUSSION}

With the triexponential analysis, three lifetimes $\left(\tau_{1,2,3}\right)$ and intensities $\left(I_{1,2,3}\right)$ were obtained. The most valuable information comes from the decay of quasi-free $\mathrm{e}^{+}\left(\tau_{2}\right)$ and of o-Ps $\left(\tau_{3}\right)$ and from the formation of o-Ps ( $\left.I_{3}\right)$. In binary AOT/isooctane solutions, a pronounced decrease in $I_{3}$ was observed for low 
concentrations, after which the decrease became less marked (Fig. 1). Similar behaviour was observed with $I_{v}$, which suggests that AOT is an efficient scavenger of $\mathrm{e}^{+}$. However, the values of $\tau_{3}$ are not so strongly affected by the AOT (3.92 - 3.72 ns) showing that the surfactant does not greatly affect the pick-off. Concerning the annihilation of quasi-free $\mathrm{e}^{+}$, values of $\tau_{2}=415 \mathrm{ps}$ for pure isooctane and $\tau_{2}=385$ ps for $2.5 \mathrm{mM}$ AOT were observed supporting the interpretation, from $I_{3}$ and $I_{V}$ measurements, that AOT is a good positron scavenger. $\tau_{2}$ also does not change significantly above $c a 2.5 \mathrm{mM}$ AOT. In contrast to a previous view [5], this suggests that, in this binary system, aggregates are not strong scavengers of $\mathrm{e}^{+}$or oPs and that monomers are still present even at high AOT concentration. These results are consistent with multiple equilibrium surfactant aggregation and with the presence of polydisperse aggregates.

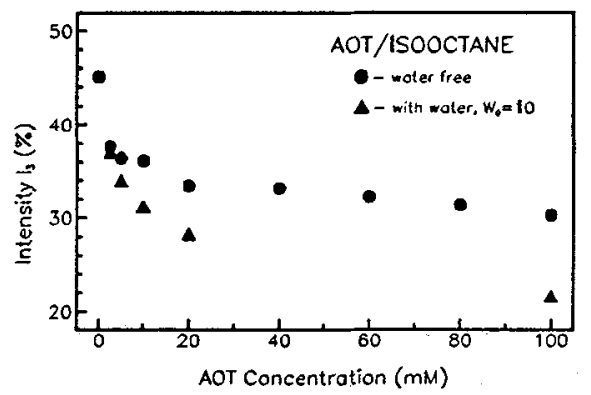

Fig. $1-\mathrm{I}_{3}$ as a function of AOT concentration

Ternary systems water/AOT/isooctane were studied (Fig. 1). At constant water/AOT mole ratio $\left(w_{0}\right)$ size of microemulsions aggregates is thought to be constant, but their number increases with concentration. With $2.5 \mathrm{mM}$ AOT, very similar behaviour was observed as with binary systems. With increasing AOT concentration only a relatively small decrease in $\mathrm{I}_{3}$ was observed. However, the decrease in $\mathrm{I}_{3}$ is greater than in the binary system, suggesting that the aggregates are also $\mathrm{e}^{+}$scavengers, and thus involved in the decrease of the Ps yield. Again, comparison of $\mathrm{I}_{3}$ values at 2.5 $\mathrm{mM}$ AOT in the presence and absence of water indicates that monomers are strong inhibitors of Ps formation. Further, although $\tau_{3}$ decreases in AOT/isooctane with increasing surfactant concentration, the values for $100 \mathrm{mM}$ AOT in isooctane with and without water $(3.68,3.72 \mathrm{~ns})$ are virtually identical and are much longer than in pure water $(1.8 \mathrm{~ns})$, showing that Ps annihilates in a water free region.

The ternary water/AOT/isooctane system was also studied at constant AOT concentration ( $100 \mathrm{mM}$ ), varying $\mathrm{w}_{0}$ values from 2 to 10 (Fig. 2). Although there is disagreement on the amount of water necessary for formation of well-defined aggregates, it is generally accepted that, at high $\mathrm{w}_{0}$ values, such aggregates exist and their volume is linearly related to $w_{0}[6]$. Figure 2 shows an initial decrease in $I_{3}$ with increasing $w_{0}$. This can be attributed to the increasing volume fraction of inverse micelles. The levelling off of $I_{3}$ at high $w_{0}$ is consistent with the idea that well defined aggregates only appear above $w_{0} \approx 5$. At constant AOT concentration, the increase in size with $w_{0}$ is counterbalanced by the decrease in aggregate concentration. $I_{V}$ measurements support the picture presented by the $I_{3}$ data. Over the range studied, $\tau_{3}$ is independent of $w_{0}$, showing that

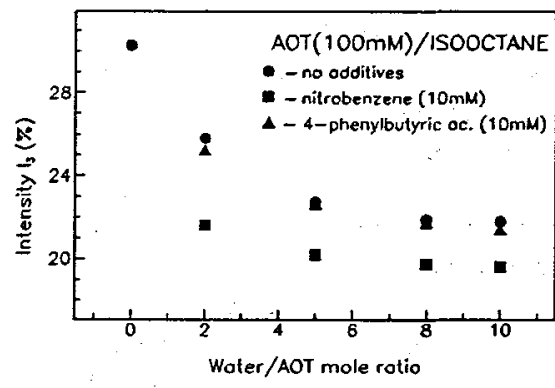

Fig. 2 Effect of increasing water/AOT mole ratio on $I_{3}$ for the ternary mixture in the presence of additives. pick-off does not appear to be dependent on the size of aggregates. This again supports the idea that o-Ps is not in a region where there is considerable contact with water. For $w_{0} \geq 5$, a value of $\tau_{2}=396$ ps is observed, which seems to be the lifetime of $\mathrm{e}^{+}$trapped in micelles.

Additives which strongly inhibit Ps formation were studied (Fig. 2). For a fixed nitrobenzene concentration $\left(10 \mathrm{mM}\right.$ ), the clear decrease in $\mathrm{I}_{3}$ shows that this additive inhibits Ps formation, although the effect becomes less marked at higher $w_{0}$ values. The same behaviour is observed with $I_{v} . \tau_{2}$ is not changed with the additive but $\tau_{3}$, although reduced, remains independent of $w_{0}$. This can be explained by the high reactivity of Ps towards nitrobenzene.

For 4-phenylbutyric acid, although $\tau_{2}$ and $\tau_{3}$ did not change significantly, $\mathrm{I}_{3}$ showed a small decrease, suggesting that, although this species can scavenge $\mathrm{e}^{+}$, in the reversed micellar medium it is not reactive towards Ps. 
The effect of nitrobenzene or sodium nitrobenzoate concentration on the ternary system was also studied (Fig. 3 and Table I). A progressive decrease in $I_{3}$ and $\tau_{3}$ was observed with increasing nitrobenzene concentration. Identical behaviour was observed with $\mathrm{I}_{\mathrm{v}}$. This confirms the high reactivity of Ps towards

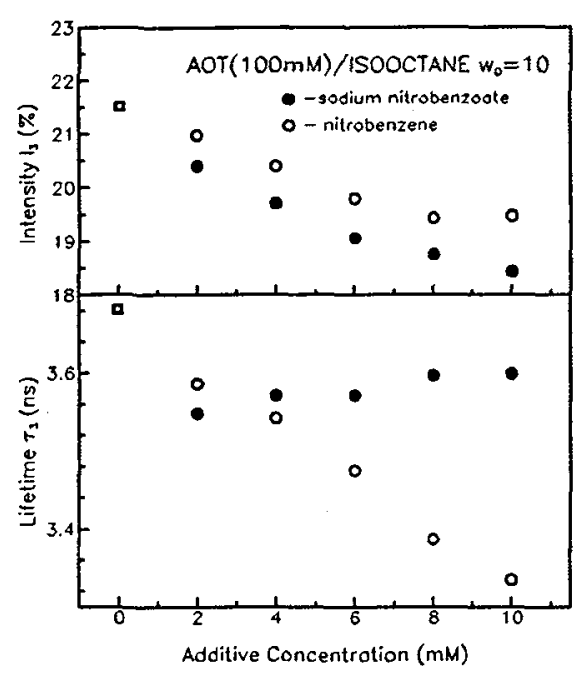

Fig. 3 - Intensities and lifetimes for different additive concentrations. The squares indicate values observed without additives. nitrobenzene. Comparison with data of nitrobenzene in isooctane suggests that the inversed micelle aggregates may favour the pick-off. $\tau_{2}$ values show no significant change. As with $\tau_{3}, I_{3}$ is more strongly affected by nitrobenzene in the ternary system than in pure isooctane. These results indicate that, apart from nitrobenzene being a strong inhibitor of Ps formation, it cannot just be in the isooctane, but must also be present in the aggregates. Since it affects Ps decay, and Ps is not affected by the water in the microemulsions, this means that nitrobenzene is at some point within the AOT layer or at the micellar interface.

Addition of sodium nitrobenzoate (which is water soluble, but insoluble in isooctane), led to a decrease in $\mathrm{I}_{3}$. The effect was more marked than that with nitrobenzene. Similar results were obtained with $I_{V}$ measurements, and suggest that sodium nitrobenzoate, which must be either in water pool or in the micellar interface, is more efficient in trapping $\mathrm{e}^{+}$than nitrobenzene.

However, although $\mathrm{e}^{+}$appears to penetrate into the micellar interior, as shown by the decreases in $I_{3}$ and $I_{v}$, the $\tau_{2}$ values does not change significantly. At present, the reason is not clear. Also the $\tau_{3}$ value does not change, even though in aqueous solutions sodium nitrobenzoate has been shown to accelerate Ps pick-off. This lack of effect on $\tau_{3}$ reinforces the view that Ps does not see the water present in the micelle.

Table I - Data from Water $\left(\mathrm{w}_{0}=10\right) / \mathrm{AOT}(100 \mathrm{mM}) /$ Isooctane with additive concentration

\begin{tabular}{|c|c|c|c|c|c|c|c|c|c|c||}
\hline \hline Conc & \multicolumn{4}{|c|}{ + Nitrobenzene } & \multicolumn{4}{c|}{ +Sodium Nitrobenzoate } \\
\hline $\mathrm{mM}$ & $\begin{array}{c}\mathrm{I}_{\mathrm{v}} \\
{[ \pm 2.0 \mathrm{mrad}]}\end{array}$ & $\begin{array}{c}\mathrm{I}_{3} \\
{[\%]}\end{array}$ & $\begin{array}{c}\tau_{3} \\
{[\mathrm{~ns}]}\end{array}$ & $\begin{array}{c}\tau_{2} \\
{[\mathrm{ps}]}\end{array}$ & $\begin{array}{c}\bar{\tau} \\
{[\mathrm{ns}]}\end{array}$ & $\begin{array}{c}\mathrm{I}_{\mathrm{v}} \\
{[ \pm 2.0 \mathrm{mrad}]}\end{array}$ & $\begin{array}{c}\mathrm{I}_{3} \\
{[\%]}\end{array}$ & $\begin{array}{c}\tau_{3} \\
{[\mathrm{~ns}]}\end{array}$ & $\begin{array}{c}\tau_{2} \\
{[\mathrm{ps}]}\end{array}$ & $\begin{array}{c}\bar{\tau} \\
{[\mathrm{ns}]}\end{array}$ \\
\hline 0 & 0.3493 & 21.6 & 3.68 & 396 & 1.09 & 0.3493 & 21.6 & 3.68 & 396 & 1.09 \\
\hline 2 & 0.3489 & 21.0 & 3.59 & 396 & 1.05 & 0.3479 & 20.4 & 3.55 & 395 & 1.02 \\
\hline 4 & 0.3478 & 20.4 & 3.54 & 397 & 1.02 & 0.3469 & 19.7 & 3.57 & 396 & 1.00 \\
\hline 6 & 0.3469 & 19.8 & 3.48 & 396 & 0.99 & 0.3459 & 19.1 & 3.57 & 396 & 0.98 \\
\hline 8 & 0.3462 & 19.4 & 3.39 & 396 & 0.96 & 0.3450 & 18.8 & 3.60 & 395 & 0.98 \\
\hline 10 & 0.3459 & 19.5 & 3.34 & 395 & 0.95 & 0.3445 & 18.4 & 3.60 & 395 & 0.97 \\
\hline
\end{tabular}

\section{REFERENCES}

/1/ VASS,S, Struct. Chem. vol.2 (1991) (167)375, and references therein.

12/ ISRAELACHIVILI,J.N, "Intermolecular and Surface Forces", Academic Press London 1989.

13/ ACHE,H.J, in "Positron and Positronium Chemistry", ed. D.M.Schrader and Y.C.Jean, Studies in

Physical and Theoretical Chemistry, 57, pp. 318, Elsevier, NY, 1988.

14/ KIRKEGAARD,P, ELDRUP,M, MOGENSEN,O.E and PEDERSON,N.J, Comp. Phys. Comm, 21 (1981) 307.

15/ ALFASSI,Z.B and ACHE H.J, J.Phys. Chem. 88 (1984) 4347.

/6/ IZQUIERDO,C, MOYA,M.L, USERO,J.L, CASADO,J, Mon. fur Chem. 123 (1992) 383. 